As a simple design the poster is easy reproducible and using web based analysis tools, monitoring the use is simple, providing useful data on patient engagement, and what information is being accessed. Clinical notes were re-audited over a 6 month period to observe sustained improvement.

Results Since implementation there has been an overall increase of patients receiving documented written and verbal discharge advice to $85 \%$, with an average of 11 clicks of electronic discharge information leaflets a day (as of 19th October 2019).

There was a $22 \%$ reduction in unplanned attendances to the paediatric emergency department over the same time period.

There has been multiple positive feedback, including via social media from both clinicians and patients about the ease of use and quality of information provided.

Other benefits include a rapid way of providing information with a potential reduction in physical leaflets needed, with resource and cost savings to services.

Conclusions The QR poster provides an innovate method of providing relevant and accessible patient information leaflets to patients with patient service experience and potential resource reduction benefits.

\section{G234(P) THE SAFE ADMINISTRATION AND AVAILABILITY OF INTRAMUSCULAR ADRENALINE FOR PAEDIATRIC ANAPHYLAXIS - QUALITY IMPROVEMENT THROUGH SIMULATION}

N Richens, B Miguras. TR, Trainee Research Association for Paediatric Emergency Medicine, UK

\subsection{6/archdischild-2020-rcpch.201}

Introduction Intramuscular adrenaline is a time-critical emergency treatment in the acute management of paediatric anaphylaxis. A low fidelity simulation project was carried out to identify the relative safety of existing emergency kit adrenaline vials compared to pre-filled adrenaline autoinjectors in the management of a simulated anaphylaxis in a 5 year old male.

Method A low fidelity simulation consisting of a paediatric registrar and a paediatric staff nurse familiar with their departments were carried out under timed conditions. 12 Paired trials were carried out across two centres in paediatric emergency departments, paediatric admission units, and paediatric inpatient wards. Each paired trial occurred during the same shift with the same staff. The time from the medical request for a defined dose of $0.15 \mathrm{ml}$ of $1: 1000$ adrenaline to be provided at the bedside in a syringe with an IM needle attached was recorded. Errors in the initially provided dose were immediately identified and the time required for the provision of the correct dose measured.

Results The mean time to completion of the simulation for the emergency kit arm was 138.3 seconds (range 96 - 223 seconds) compared to 33.6 seconds (range 17 - 54 seconds) for the adrenaline autoinjector arm. Incorrect doses of adrenaline were provided to the clinician in 2 of 6 emergency kit simulations. There were no dosing errors in the autoinjector arm. In all cases the nursing staff reported preferring the autoinjector method.

Conclusion This study provides clear evidence for the improved safety of adrenaline autoinjectors over emergency kits in the hospital management of paediatric anaphylaxis. The increased time for the emergency kit arm may partly reflect the availability of various components (vials, syringes, filter and IM needles) in different clinical settings. The significant risk of errors as a consequence of human factors in emergency drug preparation is already well-known and reflected in this study. Following these results, paediatric adrenaline autoinjectors have been adopted universally across both sites. The cost consideration of autoinjectors over adrenaline vials, particularly variation in expiry rate, is important when considering departmental policy change. However, the safety concerns were considered to outweigh the increased cost in these centres.

\section{G235(P) HIGH FREQUENCY OSCILLATORY VENTILATION IN PEDIATRIC PRACTICE: A SINGLE CENTER EXPERIENCE}

R Gabr, M Nishil, J Zahraa. PICU, King Fahad Medical City, Riyadh, Saudi Arabia

\subsection{6/archdischild-2020-rcpch.202}

Introduction High frequency oscillatory ventilation (HFOV) is as a rescue mode of ventilation however, it did not show benefit in many cases.

Objectives To identify which patient are best treated with HFOV and which ones will not improve or deteriorate, and the best way of selection and adjustment of HFOV settings in the first 48 hours.

Study Design: Retrospective observational study.

Settings: One tertiary level PICU.

Subjects: 207 patients under 18 years who were admitted to PICU with respiratory failure and switched to (HFOV) in the period between September 2010 till December 2017.

Intervention: None

Results HFOV was used 241 times. 34 cases were excluded and 207 HFOV episodes were analyzed. The median age was 12 (4, 48 IQR) months with 52.7\% males. Etiology of respiratory failure was classified as diffuse alveolar disease (DAD) and small airway disease (SAD). The most common cause of respiratory failure was ARDS, 100 (48\%) cases then pneumonia, $34(16.4 \%)$ cases, two cases of asthma and 8 with bronchiolitis. Majority had an underlying chronic illness (87.4\%). Oxygenation failure was the most common reason for HFOV (77.3\%).

Over the first 48 hours there was a significant improvement in oxygenation but faster in ventilation and acid base status. Variables that were associated with 48 hours mortality were shorter duration of $\mathrm{CMV}$, lower $\mathrm{pH}$, not using $\mathrm{NMB}$ agents, sepsis, high vasopressor index and hypotension just before switching to or within the 48 hours of HFOV. 48 hours mortality was significantly higher in patients with hematological malignancies (OR 8.73 (1.58-48.06), $p=0.013$ and in the poorly identified group that did not fit into DAD or SAD categories when compared with ARDS (OR 2.73 (1.13$6.58), p=0.030)$. Conventional ventilation settings were not statistically significant between surviving vs non-surviving groups $(p>0.05)$.

Conclusion HFOV has a great value in improving oxygenation and ventilation when conventional mode fails. The underlying disease seems to have a significant impact on the outcome and immune function and malignancies are the main categories. Hemodynamic instability and sepsis are major contributors to worse short-term outcome. 